\title{
Keynote Speaker
}

\section{The Secure Role Based Mission System (RBMS)}

\author{
Eduard Babulak ${ }^{\mathrm{a}, \mathrm{b}, *}$ \\ ${ }^{a}$ Institute of Technology and Business, Czech Republic \\ ${ }^{\mathrm{b}}$ Maharishi University of Management, USA \\ *Corresponding Author: eduardbabulak@ hotmail.com
}

\begin{abstract}
Given the current and future development of computerization and information communications infrastructures, the organizations are facing more and more challenges how to secure their information asset and information flow within the organization and globally via Internet. The ubiquitous access to Internet introduces new challenges for secure access control systems providing access authorization at any-time, from-anywhere and by anyone.

The author addresses the current state of the art and future developments in Internet, Smart Cyberspace, Cyber Security, while illustrating a secure web system, which authenticates the authorized users according to their level of trust, while providing them with proper access to a specific task(s) or assignments(s) within the organization.

The author presents an enhanced Role Based Mission System (RBMS) which extends the common Role Based Access System (RBAC) models while including user activation and usage hierarchies based on the user authentication and authorisation. The system keeps track of logging, while back-tracking the recent activities performed by the authorized personnel accessing on-site or remotely the online resources via web interface. The RBMS extends RBAC's model while facilitating alternative ways to distinguish between user authentication access activation and user's access authorization hierarchies.

While being consistent with the ANSI RBAC standard, the RBMS model provides secure access to online resources based on user's specific roles within the organisation via secure web-interface. The RBMS provides a secure web application that facilitates a restricting account access to authorized users only.

Main contribution of RBMS system is to secure any government, military or business organization information systems. The author promotes further research and development and collaborative research teams in the field of studies.
\end{abstract}

